\title{
Una Aproximación a la Cuantificación de la Controlabilidad de Estado
}

\author{
Juan C. Calderón, Lina M. Gómez y Hernán D. Alvarez \\ Facultad de Minas, Universidad Nacional de Colombia. Grupo de Investigación en Procesos Dinámicos - \\ KALMAN, Carrera 80 No 65-223, Medellín, Colombia. (e-mail: jccalde0@unal.edu.co; limage@unal.edu.co; \\ hdalvare@unal.edu.co)
}

Recibido Jun. 25, 2014; Aceptado Ago. 28, 2014; Versión final recibida Sep. 11, 2014

\begin{abstract}
Resumen
En este artículo se propone un índice numérico para cuantificar la controlabilidad de estado para sistemas no lineales suaves y continuos en el tiempo. Con base en la información contenida en los conjuntos alcanzable y controlable, se define el conjunto reversible en un tiempo t para un sistema dado. Se propone una medida del tamaño y de la forma del conjunto reversible en un espacio $n$-dimensional, las cuales permiten cuantificar la controlabilidad de estado en una escala de cero a uno, y se definen procedimientos de cálculo adecuados. Se calcula el índice de controlabilidad para tres casos de estudio verificando así su correspondencia con la controlabilidad real. La inclusión del factor tiempo y las restricciones en las acciones de control para el análisis de controlabilidad de sistemas no lineales resulta ser una herramienta útil para la implementación de sistemas de control.
\end{abstract}

Palabras clave: controlabilidad de estado, alcanzabilidad, reversibilidad, sistemas no lineales, teoría de conjuntos

\section{An Approach to Quantifying State Controllability}

\begin{abstract}
In this paper a numerical index for quantifying state controllability for continuous and smooth nonlinear systems is proposed. With information obtained from the reachable and controllable sets, a reversible set at time $t$ for a given system is defined. Size and shape measurements of a reversible set in an $n$-dimensional space are proposed allowing state controllability on a scale from zero to one. Procedures to compute such indexes are detailed. State controllability index is computed for three study cases verifying its correspondence with real controllability. The inclusion of time and admissible input constraints for state controllability analysis of nonlinear systems showed to be a useful tool for real control system implementation.
\end{abstract}

Keywords: state controllability, reachability, reversibility, nonlinear systems, set theory 


\section{INTRODUCCIÓN}

Controlabilidad es la propiedad verificada a cada sistema antes de pensar en diseñarle un sistema de control. Más aún, es uno de los conceptos fundamentales en la matemática de la teoría de control (Sontag, 1998). Prueba de tal importancia en la ingeniería de procesos y de control, es que cada libro de texto básico de esas áreas dedica alguna sección a este tema. De manera general, controlabilidad es la habilidad para conducir un sistema a cualquier lugar de su espacio de estados usando las entradas manipuladas disponibles. Este artículo utilizala definición dada por Kalman (1960) para la controlabilidad de estado desde mediados del siglo pasado. Esa definición postula la controlabilidad de estado como una propiedad binaria, es decir, un sistema tiene la propiedad o no la tiene. Hoy en día esta limitación para la controlabilidad no ha sido superada. La otra limitación en los resultados de Kalman sobre controlabilidad es que sólo es aplicable a sistemas lineales. Bertsekas (1972),Hermann y Martin (1977), Bravo et al. (2005), Gómez (2009), entre otros, han trabajado en un análogo no lineal para esta propiedad, aunque una solución analítica aún es exclusiva de los sistemas lineales y los sistemas no lineales afines con la entrada (Sontag, 1998).

Trabajos concernientes a la controlabilidad de estado tratan de caracterizar regiones donde se asegure una controlabilidad local (Eising, 1984; Cardetti y Gordina, 2008; Swanand et al., 2012). Estos trabajos siguen limitándose a sistemas lineales, como las propuestas de Bravo et al. (2005) y Kerrigan(2000) en sus trabajos en control predictivo basado en modelo. Respondek (2005) introduce lacontrolabilidad bajo restricciones de no negatividad para cierto tipo de sistemas lineales de segundo orden, correspondientes a sistemas de control reales. Recientemente, Yurtseven et al. (2013) propusó las condiciones suficientes y necesarias para la controlabilidad de algunos sistemas continuos a tramos, además de presentar una revisión corta pero general sobre el desarrollo de la controlabilidad desde el trabajo de Kalman. Para los procesos químicos por lotes, como lo expone el trabajo de Gómez et al. (2012), el concepto de controlabilidad de estado resulta limitado debido a las no linealidades y el carácter irreversible. Las aproximaciones lineales para verificar la controlabilidad de estado no resultan suficientes debido a que este tipo de sistemas son de carácter fuertemente no lineal (Seider et al., 1990). El problema se intensifica si se tiene en cuenta que las tendencias actuales de diseño indican que el análisis de controlabilidad debe integrarse desde las primeras etapas del problema de diseño de procesos químicos (Perkins y Wong, 1985). En este artículo se propone una solución para el problema de la controlabilidad de estado para los sistemas no lineales, tal como se presenta en los modelos de plantas químicas.

Considere un sistema dinámico no lineal invariante en el tiempo representado de manera general por:

$\Sigma: \dot{\boldsymbol{x}}(t)=\boldsymbol{F}(\boldsymbol{x}(t), \boldsymbol{u}(t), \boldsymbol{\theta})$

Donde $\boldsymbol{x} \in \boldsymbol{X} \subseteq \mathbb{R}^{n}$ es el vector de estados, $\boldsymbol{u} \in \boldsymbol{U} \subseteq \mathbb{R}^{m}$ es el vector de entradas manipuladas admisible y $\boldsymbol{\theta} \in \boldsymbol{\Theta} \subseteq \mathbb{R}^{q}$ representa los parámetros del sistema. $\boldsymbol{F}$ es un campo vectorial $\boldsymbol{F}: \boldsymbol{X} \times \boldsymbol{U} \rightarrow \boldsymbol{X}$ con componentes, $f_{i}(\because, \cdots) i=1, \cdots, n$ continuas y Lipschitz en un intervalo $\tau=(\sigma, \sigma+T)$. Luego,

$\boldsymbol{x}(t)=\phi(t, \sigma, \boldsymbol{x}(\sigma), \boldsymbol{u}(\cdot)) \forall t \in \tau$

representa la evolución dinámica del sistema (1) en untiempo $t$, con estado inicial $\boldsymbol{x}(\sigma)$, con $\boldsymbol{u}(\cdot) \in \boldsymbol{U}^{\boldsymbol{\tau}}$ como la función que describe la entrada exógena al sistema durante el intervalo $\tau$. $\phi$ es el mapa de transición del sistema (1).

Debe ser claro para el lector que los procedimientos presentados en este artículo tratan de verificar controlabilidad de estado de manera local. En general para los sistemas no lineales, la controlabilidad de estado no se puede garantizar de manera global. Sontag (1998) presenta una discusión amplia sobre las limitaciones para verificar la controlabilidad global de estado en sistemas no lineales, el cual sigue siendo un problema abierto.

\section{GEOMETRÍA DIFERENCIAL}

Esta sección detalla la manera de verificar la controlabilidad de estado para sistemas no lineales usando herramientas de la geometría diferencial. Las pruebas de las expresiones matemáticas están más allá de la intencionalidad de este artículo. Si el lector está interesado, Sontag (1998) presenta en detalle tales sustentos. Un sistema como el mostrado en (1) se denomina afín con la entrada si puede ser escrito de la forma:

$\dot{\boldsymbol{x}}=g_{0}(\boldsymbol{x})+u_{1} g_{1}(\boldsymbol{x})+\cdots+u_{m} g_{m}(\boldsymbol{x})$ 
La Figura 1 muestra el algoritmo de cálculo para verificar la controlabilidad de estado de sistemas como (3), usando geometría diferencial. Note que aunque muchos sistemas físicos pueden ser escritos de la forma afín con la entrada, este método no es útil para todas las clases de sistemas no lineales descritos en la Sección 1. Otro punto de interés es la complejidad asociada con el cálculo simbólico necesario para caracterizar el álgebra de Lie $\left(\mathcal{A}_{L A}\right)$ asociada al sistema. Día a día los modelos matemáticos son más complejos y grandes, luego resulta importante pensar en la utilidad de estas herramientas aplicadas a los actuales problemas industriales, más que sólo visualizar la aplicabilidad a problemas académicos.

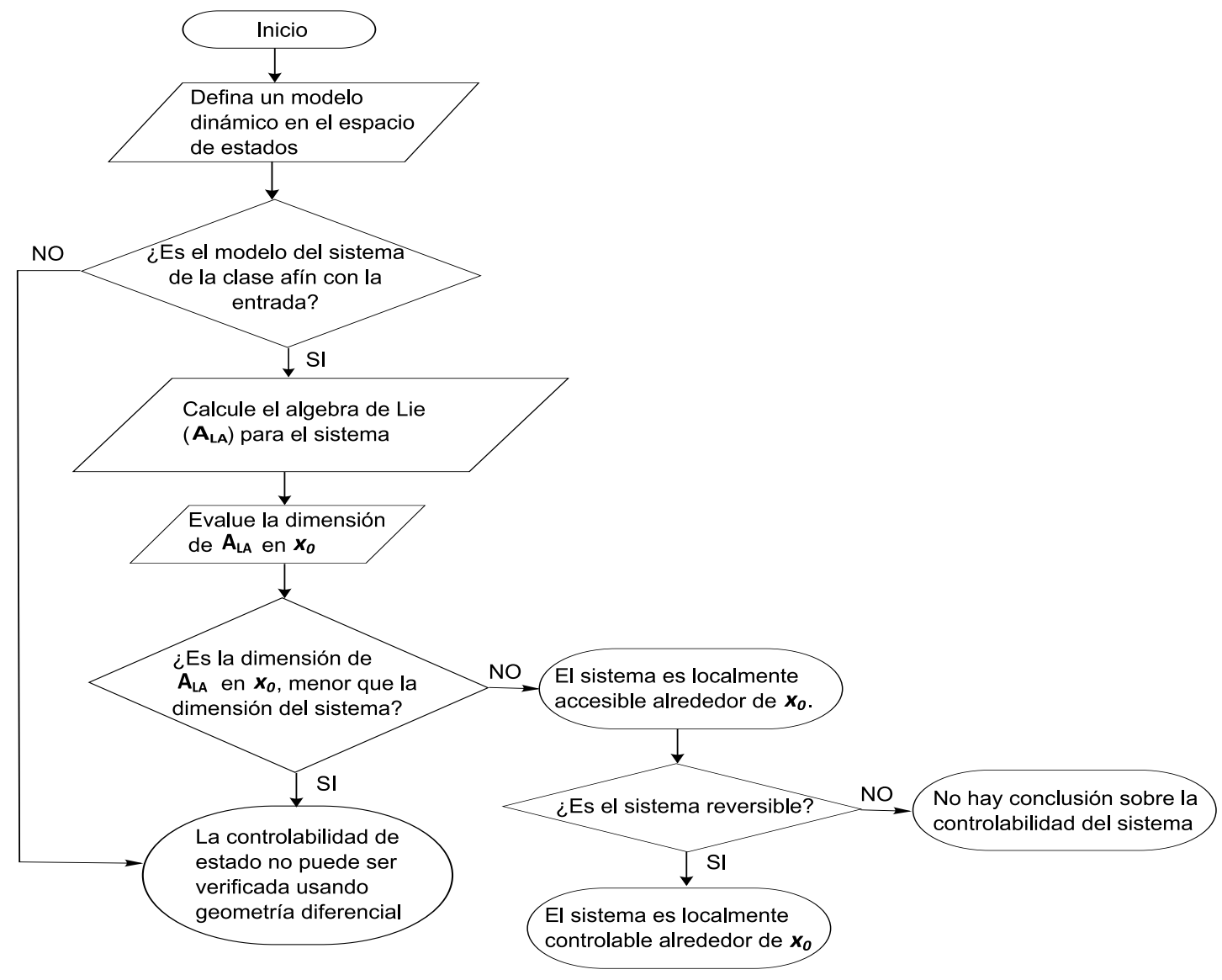

Fig. 1: Algoritmo para verificar la controlabilidad de estado usando geometría diferencial.

\section{Teoría de conjuntos y algoritmos aleatorizados}

En esta sección se combinan herramientas de la teoría de conjuntos y de los algoritmos aleatorios para determinar exitosamente la controlabilidad de un sistema no lineal de interés. Esta aproximación depende fuertemente del cálculo de los conjuntos alcanzable y controlable (Bertsekas, 1972; Sontag, 1998). El conjunto alcanzable en tiempo $T$ desde $\boldsymbol{x}_{\mathbf{0}}\left(\mathcal{R}^{T}(\boldsymbol{x})\right)$ es el conjunto de todos los puntos en el espacio de estados que el sistema puede alcanzar en tiempo $T$, bajo las acciones de control admisibles, partiendo desde $\boldsymbol{x}_{\mathbf{0}}$. El conjunto controlable a $\boldsymbol{x}_{\mathbf{0}}$ en tiempo $T(\mathcal{C}(\boldsymbol{x})$ )es el conjunto de todos los puntos desde donde el sistema puede ser conducido a $x_{0}$ en un tiempo $T$, usando las entradas de control admisibles. Los sustentos teóricos y los procedimientos de cálculo son presentados en el trabajo de Fishman (1999) y Gómez (2010) respectivamente. La Figura 2 ilustra estos dos conjuntos.

Considere un conjunto alcanzable y un conjunto controlable en tiempo $T,\left(\mathcal{R}^{T}(\boldsymbol{x})\right.$ y $\left.\mathcal{C}^{T}(\boldsymbol{x})\right)$ para el sistema representado por (1), alrededor de $\boldsymbol{x}$, el conjunto débilmente reversible es

Definición 1. El conjunto débilmente reversible alrededor de $x$ en tiempo $T$ está dado por $\Omega^{T}(\boldsymbol{x})=\mathcal{R}^{T}(\boldsymbol{x}) \cap$ $\mathcal{C}^{T}(\boldsymbol{x})$. 


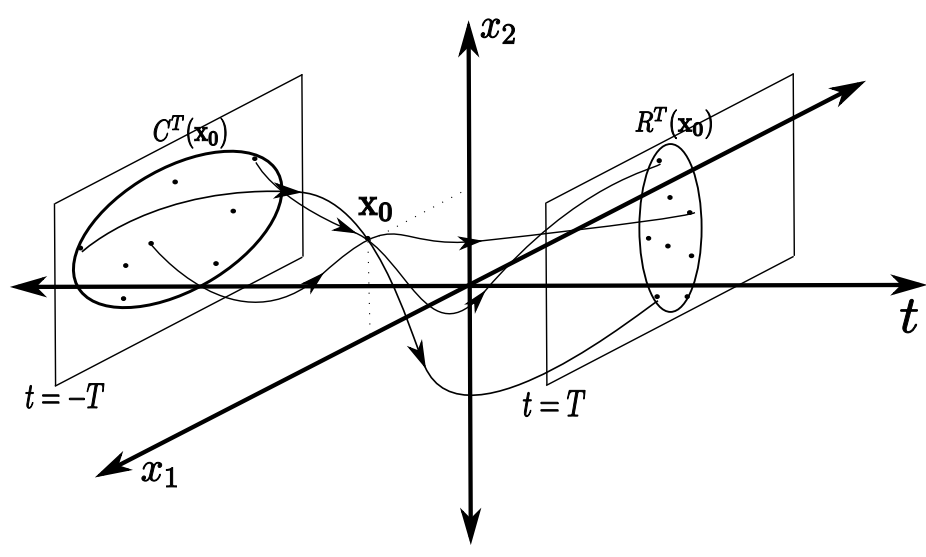

Fig. 2: Conjuntos Controlable a $x_{0}$ y Alcanzable desde $x_{0}$ en tiempo $T$.

El algoritmo de cálculo para verificar la controlabilidad de estado para un sistema no lineal usando algoritmos aleatorios y teoría de conjuntos es como sigue: i) calcular los conjuntos alcanzable, controlable y reversible, ii) Preguntar si es la dimensión del conjunto reversible menor que la dimensión del espacio de estado del sistema, iii) en caso afirmativo se concluye que el sistema no es localmente controlable alrededor de $\boldsymbol{x}_{\mathbf{0}}$, iv) en caso negativo se concluye que el sistema es localmente controlable alrededor de $\boldsymbol{x}_{\mathbf{0}}$.

\section{Cuantificación de la controlabilidad de estado}

Como se vio arriba, ninguna de las dos aproximaciones al cálculo de la controlalibidad permite cuantificar que tan controlable es un sistema. Solo permiten detectar si la propiedad existe o no existe. Representar la controlabilidad de estado de un sistema mediante conjuntos, como se presentó en la Sección 2.2, es el primer paso para asignar una métrica a la controlabilidad. Intuitivamente resulta más sencillo asignar una medida a un conjunto que a un término abstracto como la controlabilidad de estado. Debe aclararse que todos los conjuntos deben definirse en un sistema de coordenadas normalizado (hipercubo $\left[\begin{array}{ll}0 & 1\end{array}\right]^{n}$ ), con el fin de evitar deformaciones causadas por diferencias en los órdenes de magnitud en los ejes.

Note que la representación en el espacio de estado no es única. Mediante la teoría de las realizaciones, diferentes conjuntos de variables pueden establecerse como variables de estado para el mismo sistema. Obviamente, cada conjunto tiene características particulares de controlabilidad, asociadas con la medida o estimación de sus estados. Por su parte, el modelado basado en primeros principios es ampliamente usado en ingeniería de procesos como método directo para obtener un modelo en el espacio de estados. La interpretabilidad física, propia de estos modelos, resulta conveniente para redefinir el dominio de $\boldsymbol{x}$ de una manera más acorde a la realidad (Ruiz y Alvarez, 2011).

Como se ha visto, aunque existen ya dos maneras para determinar la controlabilidad en el espacio de estado, ninguno de los dos permite cuantificarla. Lo que sigue, presenta una manera de hacer tal cuantificación. En primer lugar es necesario restringir el espacio de estados de manera que siempre haya interpretabilidad física.

Definición 2. Espacio de estados práctico en tiempo $T\left(\widehat{\boldsymbol{X}}^{T}\right)$. Considere un sistema dinámico como el mostrado en (1), un conjunto de restricciones físicas o de seguridad delimitando una región $\xi \boldsymbol{x}$ un intervalo de tiempo $\tau=(\sigma, \sigma+T)$. El espacio de estados práctico en tiempo $T$, $\widehat{\boldsymbol{x}}^{T}$, es el conjunto en $\mathbb{R}^{n}$ para el cual existe una solución para (1), cumpliendo las restricciones impuestas por $\xi$, es decir,

$\widehat{\boldsymbol{x}}^{T}=\left\{\boldsymbol{x}(t) / \exists \boldsymbol{x}_{\sigma} \in \xi \wedge u(\cdot) \in \mathcal{U}^{\tau} / \boldsymbol{x}(t)=\phi_{u(\cdot)}\left(t, \sigma, \boldsymbol{x}_{\sigma}\right) \in \xi \forall t \in \tau\right\}$

donde $\phi_{u(\cdot)}\left(t, \sigma, \boldsymbol{x}_{\sigma}\right)=\phi(t, \sigma, \boldsymbol{x}(\sigma), \boldsymbol{u}(\cdot))$. Con $T$ finito, $\tau$ resulta un intervalo finito. Como la función $\phi$ está definida en $\tau$, luego $\widehat{x}$ resulta ser un conjunto acotado. Por lo tanto las definiciones para los conjuntos alcanzable y controlable tal como aparecen en Sontag (1998), son a partir de ahora referidas a $\widehat{\boldsymbol{x}}^{T}$.

Como se mencionó en la Sección 2, el conjunto reversible contiene la información más relevante sobre la controlabilidad de estado de un sistema alrededor de un punto definido. Con esto en mente, este trabajo propone indicadores para cuantificar diferentes características de tal conjunto reversible con el fin de obtener un índice numérico continuo para la controlabilidad de estado. 
Intuitivamente, un índice apropiado para la controlabilidad de estado alrededor de un estado $\boldsymbol{x}_{0}$ debe incrementar su valor si el conjunto reversible incrementa su tamaño. El tamaño es referido a una medida en $\mathbb{R}^{n}$. Debe considerarse también que la forma del conjunto reversible en relación a $x_{0}$, incluso teniendo el mismo tamaño, da nociones de diferentes capacidades del sistema en cuanto a controlabilidad. Un sistema no tendrá iguales capacidades en cuanto a controlabilidad si su conjunto reversible es hiper-esférico con centro en $\boldsymbol{x}_{0}$, o si es hiper-elipsoidal con $\boldsymbol{x}_{0}$ ubicado cerca de su frontera. Luego la forma y la posición relativa del conjunto reversible con respecto a $\boldsymbol{x}_{0}$ representa, de alguna manera, la controlabilidad de estado del sistema. Note que la posición relativa del conjunto reversible con respecto a $\boldsymbol{x}_{0}$ determina la capacidad de rechazo de perturbaciones en todas las posibles direcciones. Considerando estos aspectos, se proponen dos factores para cuantificar la controlabilidad de estado.

Definición 3. Considere un sistema dinámico continuo en el tiempo como en (1) y su respectivo espacio de estados práctico en tiempo $T\left(\widehat{\boldsymbol{x}}^{T}\right)$. Asuma que $\boldsymbol{x}_{0} \in \widehat{\boldsymbol{x}}^{T}$ y $\Omega^{T}$ es su respectivo conjunto reversible en tiempo $T$. El índice de tamaño en tiempo $T, I_{\mathrm{sz} \Omega}^{T}\left(\boldsymbol{x}_{0}\right)$, de $\boldsymbol{x}_{0}$ para el sistema (1), es el cociente entre el hipervolumen $\left(H V^{*}\right)$ de $\Omega^{T}$ y el hiper-volumen de $\widehat{\boldsymbol{x}}^{T}$, esto es

$I_{\mathrm{sz} \Omega}^{T}\left(\boldsymbol{x}_{0}\right):=\frac{H V^{*}\left(\Omega^{T}\right)}{H V^{*}\left(\widehat{\chi}^{T}\right)}$

Definición 4. Considere un sistema dinámico continuo en el tiempo como en (1) y su respectivo espacio de estados práctico en tiempo $T\left(\widehat{\boldsymbol{x}}^{T}\right)$. Asuma que $\boldsymbol{x}_{0} \in \widehat{\boldsymbol{x}}^{T}$ y que su respectivo conjunto reversible $\Omega \subset \widehat{\boldsymbol{x}}^{T} \subset$ $\mathbb{R}^{n}$ (calculado en tiempo $T$ ). Sea $\partial \Omega$, la frontera topológica de $\Omega$ y $D$ una función que mapea un punto en $\mathbb{R}^{n}$ en la frontera $\partial \Omega$ a su distancia euclidiana con respecto a $\boldsymbol{x}_{0}$, es decir: $D: \boldsymbol{x}_{0} \in \mathbb{R}^{n} \times \partial \Omega \in \mathbb{R}^{n} \rightarrow \mathbb{R}^{\text {. El }}$ índice de forma en tiempo $T, I_{\mathrm{sh} \Omega}^{T}\left(\boldsymbol{x}_{0}\right)$, se define como la relación entre la desviación estándar de las distancias de una hiperesfera centrada en $x_{0}$ a su centro y la desviación estándar de las distancias de cada punto de la frontera del conjunto reversible a $x_{0}$.Para total correspondencia de los valores extremos se propone:

$I_{\mathrm{sh} \Omega}^{T}\left(\boldsymbol{x}_{0}\right)=1-\frac{\beta(D)}{\sqrt{n}}$

Donde $\beta(D)$ es la desviación estándar de $D$ sobre todo $\partial \Omega$ y $n$ es la dimensión del espacio de estados del sistema.

El índice de tamaño y el índice de forma combinados brindan una eficiente caracterización para la controlabilidad de estado local de un sistema dado. Ambos índices están acotados entre cero y uno, luego una buena combinación de ambos debería conservar tales cotas. Con esto en mente, el índice completo para la controlabilidad de estado se define como:

Definición 5. Dado un índice de tamaño $\left(I_{\mathrm{sz} \Omega}^{T}\left(\boldsymbol{x}_{0}\right)\right)$ y un índice de forma $\left(I_{\mathrm{sh} \Omega}^{T}\left(\boldsymbol{x}_{0}\right)\right)$ para el conjunto controlable en el espacio de estado en tiempo $T$, el índice de controlabilidad de estado en tiempo $T, I_{\Omega}^{T}\left(\boldsymbol{x}_{0}\right)$ se calculará como:

$I_{\Omega}^{T}\left(x_{0}\right)=I_{\mathrm{sz} \Omega}^{T}\left(x_{0}\right) \cdot I_{\mathrm{sh} \Omega}^{T}\left(x_{0}\right)$

Generalmente no es posible calcular analíticamente $I_{\mathrm{sz} \Omega}^{T}\left(\boldsymbol{x}_{0}\right), I_{\mathrm{sh} \Omega}^{T}\left(\boldsymbol{x}_{0}\right)$ y por lo tanto $I_{\Omega}^{T}\left(\boldsymbol{x}_{0}\right)$. Esto se debe a que sólo unas pocas clases de sistemas de ecuaciones no lineales pueden ser resueltos de manera analítica. Es así como los algoritmos aleatorizados resultan ser una herramienta útil, particularmente el método de integración de Monte Carlo.

El cálculo del índice de tamaño, una vez que el conjunto está representado como una nube de puntos normalizada, es decir, que esté contenida en el hiper-cubo [ $\left[\begin{array}{ll}0 & 1\end{array}\right]^{n}$, requiere:

i) Seleccionar una región $B \in \mathbb{R}^{n}$. Tal región $B$ debe contener al espacio de estados práctico del sistema. Note que si el conjunto reversible está normalizado, el hiper-cubo $\left[\begin{array}{ll}0 & 1\end{array}\right]^{n}$ resulta ser una buena elección para $B$.

ii) Muestrear uniformemente la región $B$ con un número finito de muestras $N_{B}$. $N_{B}$ está relacionado al error (e) mediante $|e| \leq 1 / N_{B}^{1 / n}$, siendo $n$ la dimensión del espacio de estados del sistema. Para más detalles se remite al lector al trabajo de Fishman (1999).

iii) Determinar la cantidad de puntos $N_{\mathcal{R e v}}$, del total de $N_{B}$, que pertenecen al conjunto reversible.Para determinar la pertenencia de cada punto a un conjunto representado por una nube de puntos se define una 
distancia mínima de pertenencia $\overline{d_{m \imath n}}=\left(\frac{1}{N_{B}}\right)^{1 / n}$. Si la distancia euclideana desde un punto $M$ a algún punto $O \in \Lambda$, siendo $\Lambda$ un conjunto cualquiera en $\mathbb{R}^{n}$, es menor que $\overline{d_{m i n}}$, el punto $M$ pertenece al conjunto $\Lambda$.

iv) Calcular el índice de tamaño mediante

$I_{\mathrm{sz} \Omega}^{T}\left(\boldsymbol{x}_{0}\right) \approx \frac{N_{\mathcal{R e v} v^{T}}}{N_{B}}$

Por su parte, para calcular el índice de forma se requiere, una vez que el conjunto está representado como una nube de puntos normalizada, realizar estos pasos:

i) Seleccionar los puntos del conjunto reversible que están situados en la frontera topológica. Para este procedimiento se usa el método de los vecinos inversos más cercanos propuesto por Xia et al. (2006).

ii) Calcular la distancia euclideana desde $\boldsymbol{x}_{0}$ a cada uno de los puntos de la frontera topológica del conjunto reversible. Denote este conjunto de distancias como $D^{*}$.

iii) Calcular la desviación estándar $(\sigma(\cdot))$ de $D^{*}$.

iv) Calcular el índice de forma mediante:

$I_{\mathrm{sh} \Omega}^{T}\left(\boldsymbol{x}_{0}\right) \approx 1-\frac{\sigma\left(D^{*}\right)}{\sqrt{n}}$

\section{CASOS DE ESTUDIO}

En esta sección se presentan algunos casos de estudio con los que se ilustrará la utilidad del índice para cuantificar la controlabilidad de estado aquí propuesto. En todos los casos se calcula el valor del índice de controlabilidad de estado y se discuten los resultados.

\section{Sistema lineal controlable}

Con el fin de iniciar con un ejemplo simple, considere un sistema completamente controlable representado por

$\left[\begin{array}{l}\dot{x}_{1} \\ \dot{x}_{2}\end{array}\right]=\left[\begin{array}{l}u_{1} \\ u_{2}\end{array}\right]$

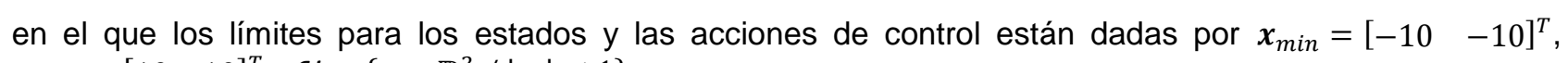
$\boldsymbol{x}_{\max }=\left[\begin{array}{ll}10 & 10\end{array}\right]^{T}$ y $U=\left\{\boldsymbol{u} \in \mathbb{R}^{2} /\left|u_{i}\right| \leq 1\right\}$.

Asuma, sin perder generalidad, que $x_{0}$ es el origen. Mediante la condición de rango de los sistemas lineales se verifica que el sistema es completamente controlable en todo $\mathbb{R}^{2}$ (Kalman, 1960). Para un tiempo definido $T=10$, el conjunto alcanzable $\mathcal{R}^{10}\left(\boldsymbol{x}_{0}\right)$ y el conjunto controlable $\mathcal{C}^{10}\left(\boldsymbol{x}_{0}\right)$ resultan ser ambos una caja unitaria en $\mathbb{R}^{2}$ centrada en el origen. Esa caja representa adecuadamente el espacio de estados práctico para este caso $\left(\widehat{\boldsymbol{x}}^{10}\right)$, luego $I_{\mathrm{sz} \Omega}^{10}\left(\boldsymbol{x}_{0}\right)=1$. De otro lado $I_{\mathrm{sh} \Omega}^{10}\left(\boldsymbol{x}_{0}\right)=0.9515$, luego $I_{\Omega}^{10}\left(\boldsymbol{x}_{0}\right)=0.9515$ para un sistema que es completamente controlable.

La Figura 3 muestra los puntos de la frontera que delimitan el conjunto reversible para el sistema (10). Es de resaltar que el índice de controlabilidad de estado es diferente de 1.0 para un sistema que es completamente controlable. Según la Definición 6, la noción de controlabilidad completa implica la misma capacidad de movimiento en todas las direcciones, esto es que el conjunto reversible esté representado por una hiper-esfera centrada en $\boldsymbol{x}_{0}$. Este sistema en particular es capaz de alcanzar las esquinas en el mismo tiempo (si no hay límites en la velocidad del elemento final de control), que es capaz de alcanzar cualquier otro punto en la frontera. Esto implica recorrer una mayor distancia en el mismo tiempo, luego no se tiene la misma habilidad de movimiento en todas las direcciones. Esta es la causa de la diferencia entre el resultado analítico y el resultado numérico en la caracterización de la controlabilidad de estado.

\section{Propiedades mecánicas}

En la Tabla 2 se muestran los resultados más relevantes de las pruebas de esfuerzo-deformación de las membranas preparadas y de la membrana comercial Nafion NRE211-10. Se evidencia que las membranas en las que predominan las funcionalidades orgánicas (sulfonada y vulcanizada), son más flexibles ya que exhiben baja resistencia a la tensión y alto porcentaje de elongación máximo (Tripathi y Shahi, 2011). 


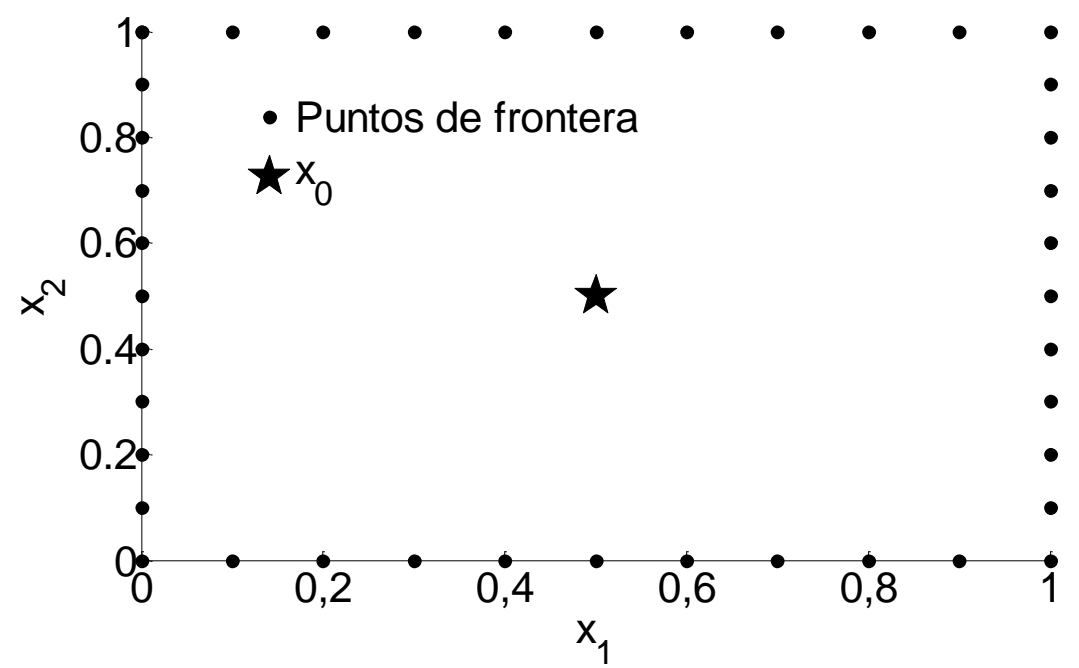

Fig. 3: Puntos de la frontera para el conjunto reversible en $T=10$ para el sistema (10)

\section{Sistema no lineal no controlable}

Considere el sistema no lineal presentado por Chen y Allgöwer (1998):

$\left[\begin{array}{c}\dot{x}_{1} \\ \dot{x}_{2}\end{array}\right]=\left[\begin{array}{c}x_{2}+u\left(\mu+(1-\mu) x_{1}\right) \\ x_{1}+u\left(\mu+4(1-\mu) x_{2}\right)\end{array}\right]$

en el que los límites para los estados y las acciones de control están dadas por: $\boldsymbol{x}_{\min }=\left[\begin{array}{ll}-10 & -2\end{array}\right]^{T}, \boldsymbol{x}_{\max }=$ $\left[\begin{array}{ll}10 & 12\end{array}\right]^{T}$ y $u=\{u \in \mathbb{R} /|u| \leq 2\}$ y $\mu=0.5$.

Considerando que $x_{0}$ es el origen, la condición de rango no permite obtener alguna conclusión sobre la controlabilidad del sistema, debido a que el rango de la matriz de controlabilidad (dimensión del algebra de Lie generada por $u$ ) es 1 , menor que la dimensión del sistema $n=2$. Calculando el conjunto reversible en tiempo $T=1$, el cual es tiempo suficiente para que el sistema se estabilice, se obtiene que $I_{\text {sz } ~}^{1}\left(\boldsymbol{x}_{0}\right)=$ 0.0150 y $I_{\mathrm{sh} \Omega}^{1}\left(\boldsymbol{x}_{0}\right)=0.9906$, luego $I_{\Omega}^{1}\left(\boldsymbol{x}_{0}\right)=0.0148$ para un sistema no controlable. En este caso el índice de controlabilidad de estado debería ser igual a 0.0 pero no lo es. La Figura 4 muestra los conjuntos calculados para el sistema (11).

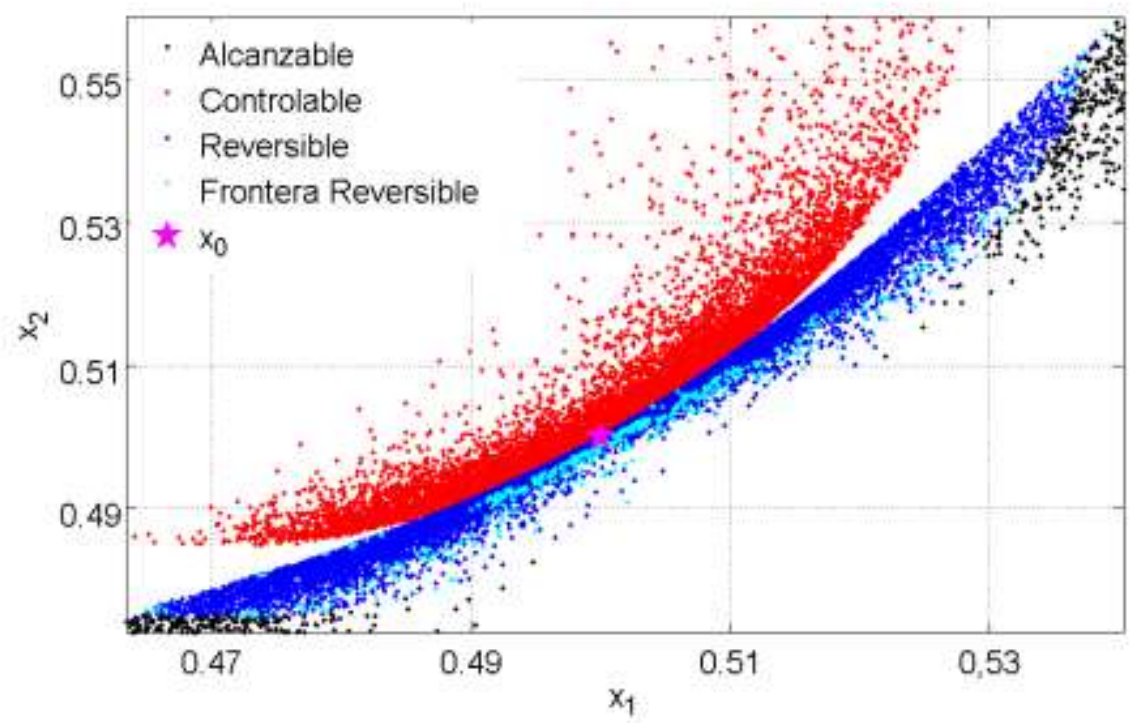

Fig. 4: Conjuntos alcanzable, controlable y reversible en $T=1$ para el sistema (11). Además los puntos que caracterizan la frontera del conjunto reversible. $\boldsymbol{x}_{0}$ es representado por una estrella. 
En la figura se puede visualizar que la intersección del conjunto alcanzable y controlable en tiempo $T=1$ es el conjunto vacío (los conjuntos alcanzable y controlable no se interceptan). Por otro lado, la misma Figura 4 muestra que los puntos que representan el conjunto reversible no son un conjunto vacío. Esto se debe a que el procedimiento numérico para calcular el conjunto reversible falla en la obtención de una representación exacta del conjunto, debido a que al definir una distancia mínima existirán puntos que cumplen la condición de pertenencia. Sin embargo, note que el índice es bastante cercano a cero, y el error cometido respecto de 0.0 es del orden de $1.4 \%$. Esta magnitud del error no es mala considerando la complejidad de los procedimientos numéricos involucrados en el cálculo del conjunto reversible. Por lo tanto es posible definir un límite arbitrario igual a $3 \%$ de error, para el cual todo valor de $I_{\Omega}^{T}\left(x_{0}\right)<0.03$ es indicativo de que el sistema tiene características que lo hacen no controlable.

\section{Sistema no lineal controlable}

Este sistema corresponde al modelo matemático de un reactor continuo de tanque agitado CSTR (del inglés Continuous StirredTank Reactor) estudiado por Bequette (2002):

$\left[\begin{array}{c}\dot{x}_{1} \\ \dot{x}_{2}\end{array}\right]=\left[\begin{array}{c}\frac{F}{V}\left(C_{A f}-x_{1}\right)-k_{0} e^{\left(\frac{-E a}{R x_{2}}\right)} x_{1} \\ \frac{F}{V}\left(T_{f}-x_{2}\right)+\frac{(-\Delta H)}{\rho C_{p}} k_{0} e^{\left(\frac{-E a}{R x_{2}}\right)} x_{1}-\frac{U A}{V \rho C_{p}}\left(x_{2}-T_{j}\right)\end{array}\right]$

con las siguientes restricciones en las acciones de control:

$u=\left\{\boldsymbol{u} \in \mathbb{R}^{2} /\left[\begin{array}{c}0 \\ 283\end{array}\right] \leq\left[\begin{array}{l}u_{1} \\ u_{2}\end{array}\right] \leq\left[\begin{array}{c}0.4 \\ 313\end{array}\right]\right\}$

Normalizando se obtiene que $\boldsymbol{x}_{0}=\left[\begin{array}{ll}0.57 & 0.29\end{array}\right]^{T}$. Por geometría diferencial el sistema es controlable. Por su parte, calculando los índices de controlabilidad de estado en tiempo $T=120$, nuevamente tiempo suficiente para todas las trayectorias calculadas se estabilicen, se obtiene $I_{s z \Omega}^{120}\left(\left[\begin{array}{ll}0.57 & 0.29\end{array}\right]^{T}\right)=0.1386$ y $I_{s h \Omega}^{120}\left(\left[\begin{array}{ll}0.57 & 0.29\end{array}\right]^{T}\right)=0.9227$, luego $I_{\Omega}^{120}\left(\left[\begin{array}{ll}0.57 & 0.29\end{array}\right]^{T}\right)=0.1279$. Las Figuras $5 \mathrm{Y} 6$ muestran los diferentes conjuntos calculados para el sistema (12) y como estos se superponen.

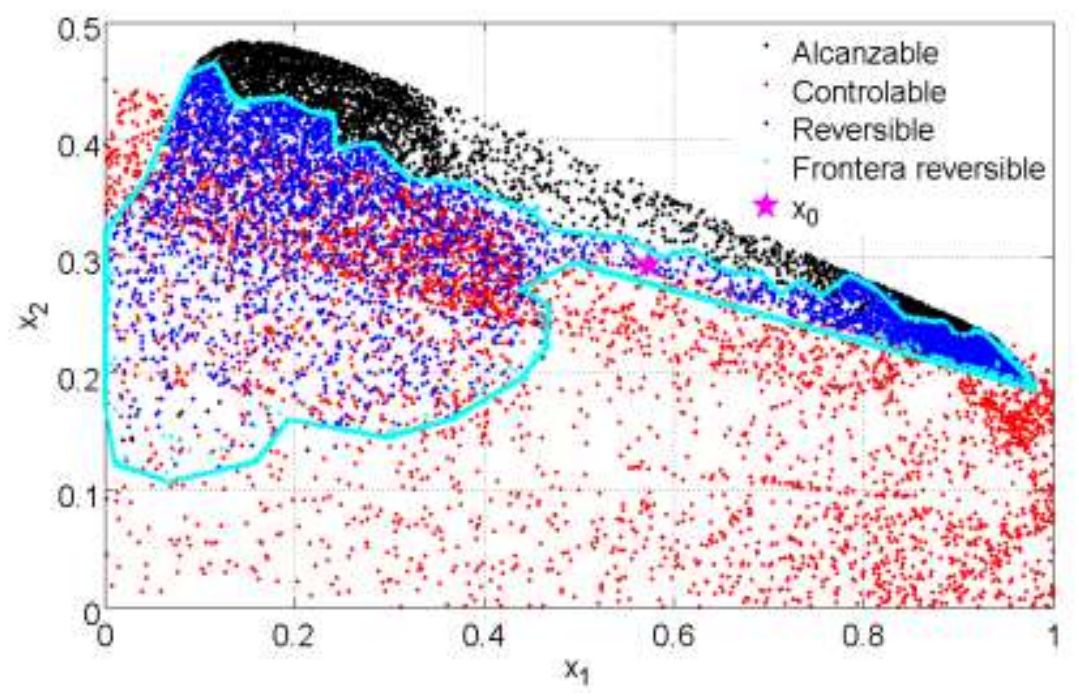

Fig. 5: Conjuntos alcanzable, controlable y reversible en $T=120$ para el sistema (12) con $V=V_{\text {nom }}$.

El índice de controlabilidad puede parecer un simple número sin sentido si se considera de manera aislada o si se define erróneamente el espacio de estado práctico. Pero si se considera el índice con fines comparativos, por ejemplo para evaluar modificaciones en plantas que afectan la controlabilidad, resulta útil puesto que permite cuantificar las variaciones en la controlabilidad de estado del sistema al cambiar el diseño. En la Figura 6 se muestran los conjuntos alcanzable, controlable y reversible para el mismo sistema (12) y de igual manera en tiempo $T=120$, con una variación de $-30 \%$ en el volumen del tanque. De manera intuitiva esto hace el reactor menos capacitivo, lo cual lo hace más fácil de manipular.

Esto se confirma con la Figura 6, donde se puede apreciar un mejor solapamiento entre los conjuntos alcanzable y controlable, haciendo mayor el tamaño del conjunto reversible. Calculando el índice de 
controlabilidad de estado para este sistema modificado se obtiene $I_{s z \Omega-2}^{120}\left(\left[\begin{array}{ll}0.57 & 0.29\end{array}\right]^{T}\right)=0.1732$ y $I_{s h \Omega-2}^{120}\left(\left[\begin{array}{ll}0.57 & 0.29\end{array}\right]^{T}\right)=0.9128$, luego $I_{\Omega-2}^{120}\left(\left[\begin{array}{ll}0.57 & 0.29\end{array}\right]^{T}\right)=0.1581$. Estos resultados evidencian un incremento del $23.6 \%$ en la controlabilidad de estado del sistema si el volumen del reactor se disminuye en $30 \%$. Con un análisis tradicional de controlabilidad sólo se podría determinar que ambos sistemas son localmente controlables alrededor de $\boldsymbol{x}_{0}$, pero sin ninguna información extra.

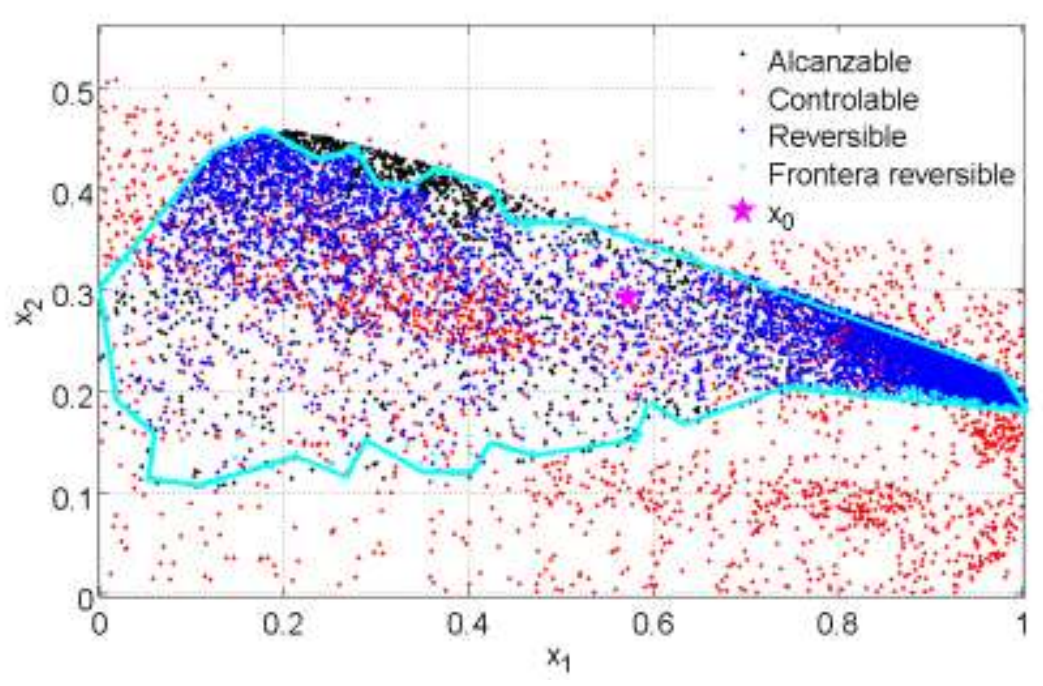

Fig. 6: Conjuntos alcanzable, controlable y reversible en $T=120$ para el sistema (12) con $V=0.7 V_{\text {nom }}$.

\section{CONCLUSIONES}

Este artículo presenta una comparación entre dos métodos para verificar la controlabilidad de estado, para sistemas no lineales continuos en el tiempo: la geometría diferencial y la teoría de conjuntos. La aproximación vía geometría diferencial resulta ser un procedimiento puramente analítico que no permite comparar la controlabilidad entre dos sistemas de naturaleza controlable. La aproximación vía geometría diferencial se reduce sólo a sistemas del tipo afín con la entrada, lo cual le resta utilidad en procesos químicos, los cuales generalmente no poseen esta estructura.

Se propuso un índice numérico para la controlabilidad de estado que permite cuantificar la controlabilidad de estado y hacer comparaciones entre diferentes sistemas controlables. Con esto en mente, resulta útil usar tal indicador para la solución de problemas tales como el diseño simultáneo de procesos, en el cual la controlabilidad de estado nunca ha sido usada de manera numérica debido a su naturaleza binaria.

\section{NOTACIÓN}

$\begin{array}{cl}\boldsymbol{x} & \text { Vector de estados } \\ \boldsymbol{u} & \text { Vector de acciones de control admisibles } \\ \boldsymbol{\theta} & \text { Vector de parámetros del modelo } \\ \mathcal{R}^{T}(\cdot) & \text { Conjunto alcanzable en tiempo } T \text { desde }(\cdot) \\ \mathcal{C}^{T}(\cdot) & \text { Conjunto controlable a }(\cdot) \text { en tiempo } T \\ \Omega^{T}(\cdot) & \text { Conjunto reversible en tiempo } T \text { desde }(\cdot) \\ \widehat{\boldsymbol{X}}^{T} & \text { Espacio de estados práctico en tiempo } T \\ I_{\mathrm{sz} \Omega}^{T}(\cdot) & \text { Índice de tamaño en tiempo } T \\ I_{\mathrm{sh} \Omega}^{T}(\cdot) & \text { Índice de forma en tiempo } T \\ I_{\Omega}^{T}(\cdot) & \text { Índice de controlabilidad de estado en tiempo } T \\ \mu & \text { Constante de modelo } \\ F & \text { Flujo volumétrico } \\ V & \text { Volumen de reacción } \\ C_{A f} & \text { Concentración de A en la alimentación }\end{array}$




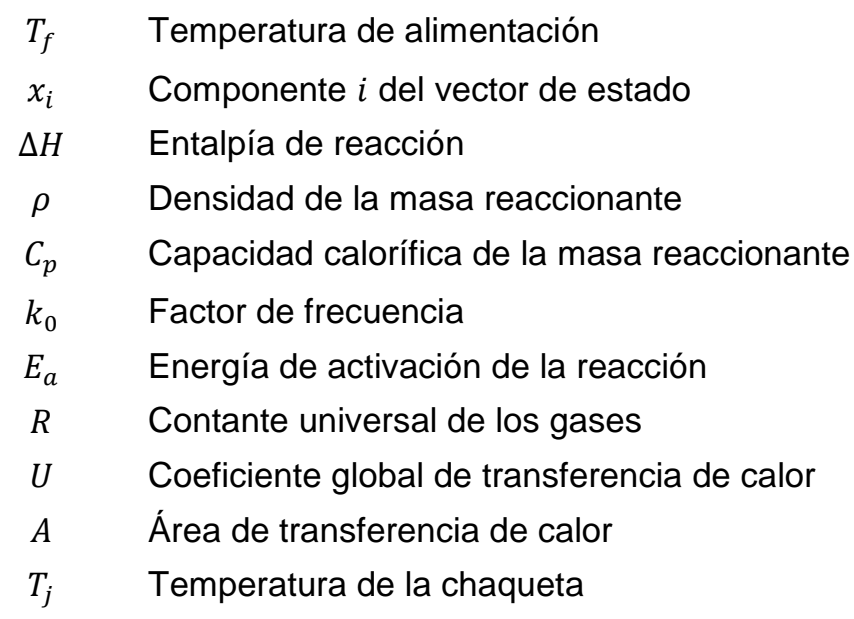

\section{AGRADECIMIENTOS}

Este trabajo fue financiado por COLCIENCIAS y su Programa de Formación Doctoral Francisco José de Caldas.

\section{REFERENCIAS}

Bequette, B.W., Behavior of a CSTR with a recirculating jacket heat transfer system, Actas del American Control Conference, 4, 3275 - 3280, Anchorage, Alaska, USA, 8 al 11 de Mayo (2002).

Bertsekas, D., Infinite time reachability of state-space regions by using feedback control, IEEE Transactions on Automatic Control 17, 604-6013, (1972).

Bravo, J. M., Limon, D., Alamo, T. y Camacho, E.F. On the computation of invariant sets for constrained nonlinear systems: An interval arithmetic approach, Automatica 41, 1583-1589, (2005).

Cardetti, F., Gordina, M., A note on local controllability on Lie groups, Systems \& Control letters, 57, 978979 (2008).

Chen, H., Allgöwer, F., Nonlinear model predictive control scheme with guaranteed stability, Automatica, 34 1205-1217, (1998).

Eising, E., Distance between controllable and uncontrollable, Systems \& Control letters, 4, 263-264 (1984).

Fishman, G.S. Monte Carlo Concepts, Algorithms, and Applications, Springer - Verlag, New York, USA (1999).

Gómez, C.A., Gómez, L.M. y Alvarez, H.D., An approach to stability and controllability analysis in batch processes using set theory methods, Actas de la conferencia del Consejo Andino de la IEEE (ANDESCON), Colombia (2010).

Gómez, L. M., Álvarez, H. D. y Botero, H. A.; Limitaciones de la Controlabilidad de Estados en los Procesos por Lotes. Información tecnolológica, 23 (5), 97-108, (2012).

Gómez, L. M. Una aproximación al control de los procesos por lotes, Tesis de doctorado, Facultad de Ingeniería, Universidad Nacional de San Juan, San Juan, Argentina, (2009).

Hermann, R. y Martin, C. Algebro-geometric and Lie-theoretic techniques in systems theory, Math Sci Press, (1977).

Kalman, R. E., On the general theory of control systems, I IFAC Congress on Automatic Control, USSR (1960).

Kerrigan, E. C., Robust constraint satisfaction: Invariant sets and predictive control, Ph.D. thesis, Dpto de ingeniería, Universidad de Cambridge, Cambridge, Inglaterra (2000).

Perkins, J. Wong, M., Assessing controllability of chemical plants, Chemical Engineering Research and Design, 63, 358-362 (1985). 
Respondek, J., Controllability of dynamical systems with constraints, Systems \& Control letters, 54, 293-314, (2005).

Ruiz, A. A. y Alvarez, H. Escalamiento de procesos químicos y bioquímicos basado en un modelo fenomenológico. Información Tecnológica, 22 (6), 33-52, (2011).

Seider, A.M.P.W.D., Brengel, D.D., Widagdo, S., Nonlinear analysis in process design. Why overdesign to avoid complex nonlinearities?, Industrial and Engineering Chemistry Research, 29, 805-818 (1990).

Sontag, E.D. Mathematical Control Theory - Deterministic Finite Dimensional Systems, $2^{\underline{a}}$ edición, SpringerVerlag, New York, USA (1998).

Swanand R.K., Pillai, H.K., Belur, M.N., Computing the radius of controllability for state space systems, Systems \& Control letters, 61(4), 327-333 (2012).

Xia, C., Hsu,W., Li, M., Beng, L., Ooi, C., Border: Efficient computation of boundary points, IEEE Transactions on Knowledge and Data Engineering, 18, 289-303, (2006).

Yurtseven, M.C.E., Heemels,W., Controllability of a class of bimodal discrete-time piecewise linear systems, Systems \& Control letters, 62, 338-344 (2013). 
